\section{Kein Rückgang von Neuralrohrdefekten}

edes Jahr wird bei etwa 5.000 Föten in Europa ein Neuralrohrdefekt festgestellt. Frauen mit Kinderwunsch wird eine Folsäuresupplementation empfohlen, die offenbar nur wenige umsetzen.

In einer populationsbasierten Studie wurde nun die Häufigkeit von Neuralrohrdefekten anhand der Daten des European Surveillance of Congenital Anomalies(EUROCAT)-Registers (19912011, 12,5 Mio. Geburten in 19 europäischen Ländern) analysiert. $11.353 \mathrm{Neu-}$ ralrohrdefekte waren registriert, die nicht im Zusammenhang mit Chromosomenanomalien standen, darunter 4.162 Anenzephalien und 5.776 Kinder mit Spina bifida. Die europäische Ge- samtprävalenz betrug 9,1/10.000 Geburten (Lebendgeburten, fötaler Tod, Schwangerschaftsabbruch wegen fötaler Anomalie). Zwar gab es im Verlauf Schwankungen, doch keinen Abwärtstrend der Fallzahlen. Unter Einbezug von Störfaktoren zeigte sich 1995-1999 eine jährliche Steigerung um 4\%, 1999-2003 eine Reduktion um 3\%, danach blieben die Zahlen stabil. Betrachtete man Anenzephalie und Spina bifida einzeln, änderte sich die Häufigkeit auch nicht wesentlich. Die Autoren merken an, dass die (fehlende) Folsäure natürlich nicht der einzige mögliche Grund für die relativ unveränderte Neuralrohrdefektrate ist. Auch Nikotinkonsum der Mutter, chro- nische Krankheiten (z. B. Diabetes) und Antiepileptika sind Risikofaktoren für Neuralrohrdefekte. Zudem müssten Veränderungen in der Bevölkerungsstruktur berücksichtigt werden. Dennoch schlussfolgern sie: Obwohl seit 20 Jahren bekannt sei, dass die Folsäuresupplementation die Neuralrohrdefektrate senke, und schwangeren Frauen zur Folsäuresupplementation geraten werde, sei es in Europa nicht gelungen, effektive Präventionsstrategien zu etablieren. Unter NutzenRisiko-Abwägung sollte man daher über eine Folsäureanreicherung von Grundnahrungsmitteln nachdenken.

Dr. Christine Starostzik

Khoshnood B. et al. Long term trends in prevalence of neural tube defects in Europe: population based study. BMJ 2015; 351: h5949

\section{Zervikale Adenokarzinome: nicht alle HPV-assoziiert}

\author{
Zervikale Adenokarzinome nehmen in manchen Regionen der Welt zu. Anders \\ als bei invasiven squamösen Zervixkarzinomen, lässt sich bei Adenokarzinomen \\ die kausale Beziehung zu humanen Papillomviren nicht immer bestätigen.
}

$E_{x}^{n+m}$ wa $10-20 \%$ aller invasiven Zervixkarinome werden durch Adenokarzinome verursacht. In manchen Ländern steigt die Inzidenz von zervikalen Adenokarzinomen im Verhältnis zu Plattenepithelkarzinomen, besonders bei jüngeren Frauen. Schätzungen gehen für 2015 von einer weltweiten Inzidenz von 56.805 zervikalen Adenokarzinomen aus. Etwa 50\% aller Fälle von zervikalen Adenokarzinomen treten in Südostasien auf, wo der $\mathrm{Zu}$ gang zur Krebsfrüherkennung limitiert ist. Während der Nachweis von humanen Papillomviren (HPV) bei squamösen Zervixkarzinomen in 99,7\% gelingt, lässt sich HPV bei Adenokarzinomen mit bisher nur bei 62-90\% der Fälle detektieren.

Die bisher größte Studie zum zervikalen Adenokarzinom wurde nun in China durchgeführt. In dieser multizentrischen Studie aus neun Regionen des Landes wurden 718 histologisch bestätigte Fälle von zervikalen Adenokarzinomen mittels hochsensitiver Breitspektrum-PCR, bei der das HPV-L1- und HPV-E6-Gen in Gewebsschnitten nachgewiesen wurde, analysiert. Jeder Fall wurde von einem Expertengremium ausführlich diskutiert und beurteilt. Bei gewöhnlichen Adenokarzinomen, adenosquamösen und neuroendokrinen Karzinomen zeigte sich durch den Nachweis von HPV-16, 18 und 45 in Gewebe und Tumorzellen eine eindeutige Assoziation. Hingegen betrug die HPV-Positivität in anderen Typen von zervikalen Adenokarzinomen nur 45\% und virale Gene ließen sich in den Tumorzellen nicht nachweisen. Für diese Fälle wurde eine genetische Ursache vermutet. Das mittlere Alter der Frauen mit zervikalen Adenokarzinomen, bei denen HPV nachgewiesen wurde, betrug 44 Jahre, im Vergleich zu 50,5 Jahre bei HPV-negativen Tumoren. Die Autoren stellen die Hypothese auf, dass bei einer Untergruppe von Adenokarzinomen epigenetische und/oder somatische genetische Veränderungen für die Karzinogenese verantwortlich sein könnten. Gestützt wird dies durch die niedrigere Rate an p16INK4a bei HPV-negativen Adenokarzinomen, was darauf hinweist, dass die HPV-E7-Expression, pRB-Sequestration und p16-Heraufregulierung nicht aktiv ist. HPV ist dann nicht mehr an der Tumorentwicklung beteiligt oder möglicherweise während der Tumorprogression eliminiert worden.

Molijn A et al. The complex relationship between human papillomavirus and cervical adenocarcinoma. Int J Cancer 2016; 138:409-16

\section{Kommentar}

Die bisherige Aussage, dass nahezu 100\% aller invasiven Zervixkarzinome durch HPV verursacht werden, muss durch die Ergebnisse dieser Studie etwas eingeschränkt werden. Immerhin werden 3-5\% der invasiven Zervixkarzinome durch HPV-negative Adenokarzinome verursacht. Somit ist für die Karzinogenese des zervikalen Adenokarzinoms von einer Heterogenität auszugehen. Die bisherigen epidemiologischen Befunde, die HPV-16, 18 und 45 für $90 \%$ der zervikalen Adenokarzinome als Ursache fanden, wurden bestätigt.

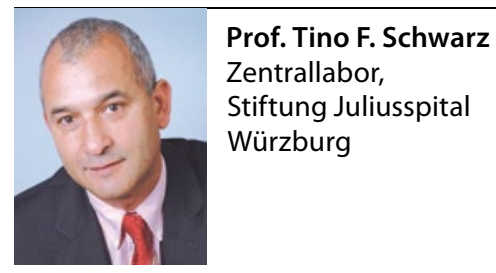

